\title{
Töten im Livestream ${ }^{1}$ \\ Facebook Live und ein neuer Invective Gaze in den Sozialen Medien
}

Verena Straub

\begin{abstract}
Der Beitrag stellt die Frage, inwiefern die Praxis des Livestreaming in den Sozialen Medien $z u$ einer neuen Konstellation herabsetzender, mitunter sogar tötender Blicke beiträgt. Mit dem Täter-Selfie vom Tatort, der Egoshooter-Perspektive des Attentäters und dem Videozeugnis aus Opfersicht rücken drei Beispiele des "Tötens im Livestream « in den Fokus, die User:innen auf ganz unterschiedliche Art und Weise affizieren und ethisch involvieren. Der sich unter den Bedingungen des Livestreaming realisierende invective gaze wird dabei als grundlegend relationales Geschehen charakterisiert, das sich durch komplexe Verflechtungen von Blicken zwischen Tätern, Opfern und Medienpublikum auszeichnet.
\end{abstract}

Livestreaming ist $\mathrm{zu}$ einer der wichtigsten Bildpraktiken in den Sozialen Medien geworden. Online-Dienste wie YouNow, YouTube Live, Periscope oder Facebook Live bieten ihren User:innen die Möglichkeit, Videos kostenlos und in Echtzeit hochzuladen, mit dem bestehenden Netzwerk zu teilen und gegenseitig zu kommentieren. Das Posten von Live-Feeds verspricht ein hohes Maß an Authentizität und Unmittelbarkeit, es suggeriert ein Gefühl des Dabeiseins und der Intimität, verdankt seine Popularität in den Sozialen Medien aber vor allem der Interaktion, die sich ebenso in Echtzeit vollzieht (vgl. Kohout 2017: 74). Das Ziel von Facebook Live, so betonte Mark Zuckerberg im Februar 2016, kurz nachdem das Feature für alle US-amerikanischen Nutzer:innen verfügbar wurde, sei es, »die persönlichste, emotionalste, roheste und instinktivste Art der Kommunikation« zu unterstützen (Honan: 2016). Dabei war es vor allem das »Rohe« dieser Kommunikationsform, das Schlagzeilen machen sollte und Facebook Live zu einem regelrechten Forum für Gewaltdarstellungen werden ließ. Seit 2016 nutzen Attentäter:innen unterschiedlichster politischer Richtungen das Feature, um ihre Taten live für ihre Follower:innen $\mathrm{zu}$ inszenieren. Aber auch Opfer von Gewalttaten - so zeigen Videozeugnisse 
von US-Polizeigewalt - setzen Facebook Live als Mittel ein, um die erlittene Gewalt in Echtzeit zu bezeugen und anzuklagen.

Ausgehend von diesen Beobachtungen stelle ich in meinem Beitrag die Frage, inwiefern das Live-Format in den Sozialen Medien eine spezifische Form der invektiven Kommunikation und Darstellung prägt. Trägt die interaktive Live-Kommunikation $\mathrm{zu}$ einer neuen Konstellation herabsetzender, in manchen Fällen sogar tötender Blicke bei? Inwiefern führen Livestreams gleichzeitig aber auch dazu, die bezeugte Gewalt für Kritik zu öffnen (wie im Fall der Black Lives Matter-Bewegung)? Welche Rolle kommt dabei den kommerziellen LivestreamAnbietern selbst als Gatekeepern der veröffentlichten Inhalte zu? Und schließlich: Welche Verantwortung geht mit dem Anklicken und Anblicken dieser in Echtzeit nachzuvollziehenden invective gazes einher? Diese Fragen werden anhand von drei Beispielen auf Facebook Live erörtert, in denen das Töten auf ganz unterschiedliche Weise ins Bild rückt. Die politischen Kontexte der drei Fälle könnten kaum weiter voneinander entfernt sein. Sie reichen von einem jihadistischen Attentat auf zwei Polizeiangehörige in Frankreich, über den rechtsextremen Anschlag auf eine Moschee in Neuseeland bis zu rassistisch motivierter Polizeigewalt in den USA. Gegenläufige Perspektiven ergeben sich aber nicht nur aus politischer Sicht. Die Videos offenbaren auch drei ganz unterschiedliche Blickkonstellationen, die auf populären Bildpraktiken im Social Web beruhen und durch das Livestreaming zusätzlich potenziert werden: Das Täter-Selfie vom Tatort, die Egoshooter-Perspektive und das Videozeugnis aus Opfersicht.

\section{1) Das Täter-Selfie vom Tatort als interaktive Praxis}

Am 13.6.2016 ermordete Larossi Abballa, ein den französischen Behörden bekannter Jihadist, den Polizisten Jean-Baptiste Salvaing mit mehreren Messerstichen vor seinem Wohnhaus im Pariser Vorort Magnanville. Anschließend stürmte der Attentäter das Haus, ermordete auch dessen Ehefrau Jessica Schneider, die ebenfalls für das Polizeikommissariat arbeitete, und nahm deren dreijährigen Sohn in Geiselhaft. Abballa drohte daraufhin der Polizei, das Haus in die Luft zu sprengen (vgl. Wiegel: 2016). Noch während sich der Attentäter mit dem Kleinkind am Tatort befand und die Polizei versuchte, mit ihm zu verhandeln, streamte er ein rund 13 Minuten langes Selfie-Video auf seinem persönlichen Facebook-Profil per LiveFunktion, in dem er eine zuvor geschriebene Botschaft auf Französisch verliest. ${ }^{2}$

2 Auch wenn das Video zusammen mit dem Account des Attentäters nach rund 11 Stunden von Facebook gelöscht wurde, sind mehrere kürzere Versionen bis heute im Internet zu finden. Die Onlineausgabe der britischen Daily Mail veröffentlichte etwa einen 37 Sekunden langen Ausschnitt. Vgl. Allen/Summers/Robinson 2016. 
Darin bekannte sich Abballa unter Berufung auf den Islamischen Staat zu den gerade verübten Taten, rühmte sich seiner Morde und drohte weitere Bluttaten des IS an. Nach Angaben des Jihadismus-Experten David Thompson, der als einer von wenigen das Video im Livestream sah, verfolgten zu diesem Zeitpunkt 98 Menschen die Ansprache des Attentäters (vgl. Stern Online 2016). Als ein Sondereinsatzkommando der Polizei das Haus bereits umstellte, bat Abballa seine »Brüder«, sie mögen für ihn zu Allah beten, dass er als »Märtyrer « sterben werde (Rubin/Blaise 2016: o.S.). Kurze Zeit später stürmten Polizeibeamte das Haus, töteten Abballa und konnten das unter Schock stehende Kleinkind lebend retten.

Der direkt in die Kamera gerichtete Täterblick und die zu weiteren Morden anstachelnde Videoansprache sind an sich nicht neu. Sowohl das auf Nachahmung angelegte Bekenntnis als auch die Selbststilisierung als Märtyrer schließen an gängige Konventionen des Videotestaments an. ${ }^{3}$ Neu ist hier jedoch, dass der Attentäter sein Video als Selfie am Tatort selbst aufnahm. Neu ist zudem das Live-Format, über das er seine Botschaft aussandte. Beide Umstände haben weitreichende Folgen für die Funktion dieses Videos und sind prägend für dessen invektives Potenzial. Im Unterschied zu anderen Videotestamenten von Selbstmordattentäter:innen, die prinzipiell vor der Tat aufgenommen und nach der Tat veröffentlicht werden, fallen Produktion und Rezeption dieses Testaments mit dem Vollzug der Tat selbst zusammen. Zwei Punkte sind hier entscheidend: Die raumzeitliche Situierung der Videobotschaft und die in Echtzeit stattfindende Interaktion zwischen Attentäter und Medienpublikum.

\section{Der situierte Blick}

Mit seiner Videoaufnahme schließt der Täter an die situative Praxis des Selfies an. Damit steht das Video in einem starken Widerspruch zum Großteil der Videotestamente des IS, die meist vor generischen Kulissen wie Flaggen und Waffen, teilweise sogar vor digital austauschbaren Bluescreens inszeniert werden und damit den Eindruck einer maximalen Ortlosigkeit vermitteln (vgl. Straub 2021; 2019). Das Selfie erhält seinen Sinn hingegen gerade durch seinen Kontext, in dem es aufgenommen wird und zu dem sich die darstellende Person ins Verhältnis setzt (vgl. Gunthert 2019: 154). Neben den sprachlich vorgetragenen Hassbotschaften und Drohungen, scheint die invektive Wirkmacht dieses Selfies allein schon in der Tatsache begründet zu liegen, dass es in der Wohnung der Ermordeten aufgenommen wurde. Im Hintergrund des verwackelten Videos sind immer wieder persönliche Gegenstände (z.B. an der Wand hängende rote Taschen) der Opfer zu sehen. Die 
leichte Untersicht, in der sich der Attentäter vor dieser Kulisse filmt, lässt ihn zugleich bedrohlich und überlegen erscheinen (Abb. 1). Auch unabhängig von der verlesenen Botschaft vollzieht die situierte Selbstdarstellung im Selfie-Modus - und insbesondere der selbstherrliche Blick in die Kamera - eine erneute Herabwürdigung der Opfer. Die Grenzüberschreitung des Mordens setzt sich in der medialen Inszenierung fort, indem der Attentäter in den Privatraum der Ermordeten eindringt und diesen zynischerweise als Schauplatz für seine Helden- und Märtyrerdarstellung wählt. Der für Selfies typische Gestus »Schaut her - Hier bin ich!« kann in diesem Fall als Gestus der Eroberung und Unterwerfung gedeutet werden. Mit seinem Selfie vom Tatort verletzt Abballa die Intimsphäre seiner Opfer und stellt diese Abwertung zugleich triumphierend zur Schau.

Abb. 1

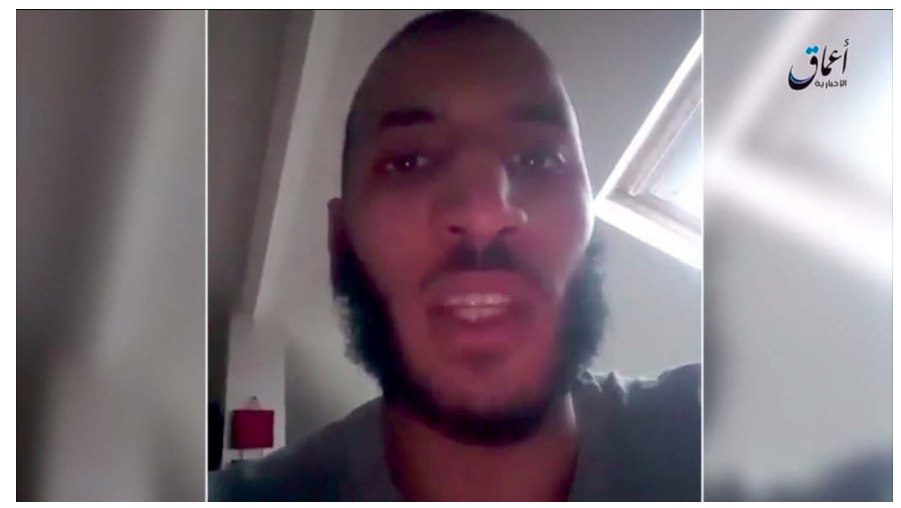

\section{Der vernetzte Blick}

Neben der räumlichen Situierung ist es aber vor allem der Livemodus, der den herabsetzenden Blick des Attentäters in eine neue - nämlich interaktive - Konstellation einbindet. Durch die Wahl der Live-Videoplattform verstärkt sich die kommunikative Funktion, die schon für unbewegte Selfies als charakteristisch hervorgehoben wurde. Sowohl André Gunthert als auch Wolfgang Ullrich verstehen Selfies im Wesentlichen als Angebote zur Interaktion (vgl. Gunthert 2019: 158). Die Schnelligkeit von Produktion und Verbreitung sowie der wechselseitige, zur Konversation anregende Austausch von Selfies rücken das Genre in die Nähe einer mündlichen Sprechkultur (vgl. Ullrich 2019: 54). Durch den Livestream wird dies zusätzlich potenziert. Statt sich an ein zukünftiges, imaginäres Publikum zu richten, zielt Abballa mit seinem Videobekenntnis auf eine Anerkennung in Echt- 
zeit, auf unmittelbare Reaktionen oder sogar konkrete Handlungsvorschläge von seinen Facebook-Follower:innen ab. An einer Stelle des Livestreams wurde dies besonders deutlich, als der dreijährige Sohn des ermordeten Paars im Hintergrund zu erkennen war und der Attentäter in die Kamera sprach: »Ich weiß noch nicht, was ich mit ihm mache«. Auch wenn die beiden Morde bereits verübt waren, so war im Moment der Videoausstrahlung der Ausgang des Attentats noch offen und das Schicksal des in Geiselhaft befindlichen Jungen noch unentschieden. Die 98 User:innen, die das Video live am Bildschirm verfolgten (vgl. Stern Online 2016), wurden augenblicklich in die Situation vor Ort involviert und zumindest prinzipiell in die Lage versetzt, mit dem Attentäter in Kontakt zu treten und damit aktiv in den Verlauf des Geschehens einzugreifen. Kommentare oder Emoticons, die während eines Livestreams auf Facebook Live gepostet werden, erscheinen in Echtzeit für alle sichtbar im Bildfeld und ermöglichen prompte Reaktionen von Seiten des Senders. Wurde die Selbstdarstellung des Attentäters durch Likes affirmiert? Gab es auch kritische Kommentare, die das Vorgehen des Täters in Frage stellten? Welchen Einfluss hatte die Echtzeitkommunikation auf die Entscheidung des Attentäters, den Sohn der Ermordeten am Leben zu lassen? Inwiefern es in diesem Fall zu einer interaktiven Situation zwischen dem Attentäter und seinem Medienpublikum kam, ist im Nachhinein nur noch schwer zu rekonstruieren. ${ }^{4}$ Allein die Möglichkeit, vom eigenen Bildschirm aus zu intervenieren, verdeutlicht jedoch, dass mit dem Betrachten eines solchen Livestreams eine gesteigerte Verantwortung einhergeht. Sobald man die Tragweite dessen begreift, was hier in Echtzeit bezeugt wird, ist ein Wegklicken, ein Wegsehen kaum mehr möglich.

Dabei ist das Blicken im Web 2.0 immer schon mit aktivem Handeln verbunden. Schon indem wir die Klickzahl eines Videos erhöhen, vor allem aber durch Praktiken des Likens, Teilens und Kommentierens wird deutlich: Ein passives Rezipieren der Bilder ist im Web 2.0 unmöglich geworden. Während häufig die ökonomische Dimension betont wird, die sich aus dieser beständigen Co-Produktion von Inhalten ergibt, also die Verschiebung von Konsument:innen zu Prosument:innen im Netz (vgl. Blättel-Mink/Hellmann 2010), drängt bei den hier diskutierten Beispielen vor allem die ethische und moralische Beteiligung des Medienpublikums in den Vordergrund: Die Frage nach der Mittäterschaft durch Anblicken und Anklicken. Es geht nicht mehr nur darum, dass die Betrachtenden zu (unfreiwilligen) medialen Augenzeug:innen und Multiplikator:innen einer Gewalttat werden. Als Teilnehmende eines auf Interaktion angelegten Livestreams werden die Follower:innen auf weitaus direktere Art und Weise zu (potenziellen) Akteuren in einem Geschehen, das vom physischen Tatort in die digitale Sphäre erweitert wird. 


\section{Der Invective Gaze als relationales Geschehen zwischen Herabsetzung und Erhöhung}

Das Konzept der Invektivität, das Herabsetzung nicht als singulären Kommunikationsakt, sondern als performatives Geschehen und relationales Geflecht von Resonanzen und Anschlusskommunikationen fasst, erscheint in diesem Zusammenhang besonders fruchtbar (vgl. Konzeptgruppe »Invektivität« 2017). Denn wie oben deutlich wurde, vollzieht sich der live übertragene invective gaze des Attentäters gerade in Relation mit den - durch Klicks und Posts - vermittelten Blicken und Reaktionen seiner Facebook-Follower:innen, die die Inszenierung des Attentäters durch unmittelbares Feedback affirmieren, unter Umständen aber auch kritisieren und ihrerseits abwerten können. Das invektive Geschehen geht also weit über die Selfie-Inszenierung des Attentäters hinaus und umfasst sämtliche - schon zeitgleich stattfindende - Resonanzen im digitalen Raum.

Invektive Kommunikation hat häufig mehrere Adressaten zugleich, kann in verschiedene Richtungen wirken und unterschiedliche Funktionen bedienen. Das zeigt auch der Livestream aus Magnanville: Der invective gaze des Attentäters ist nicht nur als Angriff gegen die von ihm angesprochenen Feinde gerichtet (die als pars pro toto für die liberale demokratische Gesellschaft stehen können), er richtet sich gleichzeitig - und vielleicht sogar primär - an seine Unterstützer und ISKontakte, die sein Selbstbild als skrupelloser Rächer und »Märtyrer« anerkennen sollen. Dass Abballa ausgerechnet Facebook Live als Arena für sein Selfie-Video gewählt hat, spielt dabei eine bedeutende Rolle. ${ }^{5}$ Bedingt durch die algorithmische Struktur der Plattform, die alle Follower:innen eines Senders oder einer Senderin per Nachricht informiert, sobald ein Livestream gestartet wird, werden Inhalte bevorzugt für den Kreis der eigenen Facebook-Freunde sichtbar. Als kommunikativer Akt zielt der invective gaze des Attentäters damit ebenso sehr auf Anerkennung aus den eigenen Reihen wie auf Einschüchterung seiner Feinde ab. Die Herabsetzung dient hier zugleich als Auszeichnung seiner kompromisslosen Hingabe für den jihadistischen Kampf. Dies wird etwa deutlich, wenn er in seinem Video sagt: »Wir [gemeint ist der IS] sind gnadenlos und herzlos... ich war gerade gnadenlos gegenüber diesem Polizisten und seiner Frau.« Je skrupelloser die Selbstdarstellung, je kaltblütiger der Blick in die Kamera, desto größer seine Hoffnung, als »Märtyrer« Arenen werden institutionell, gesellschaftlich oder medial definierte Handlungs- und Kommunikationsräume gefasst, die mit bestimmten Handlungslogiken, Aufmerksamkeitsmustern und Sprechlizenzen einhergehen und invektive Akte entscheidend prägen. Vgl. Konzeptgruppe »Invektivität« 2017: S. 13. 
des Islamischen Staats geehrt zu werden - und dies nicht nur posthum, sondern bereits während des Livestreams durch das Feedback seiner Follower:innen. ${ }^{6}$

\section{Bildkontrolle und Kontrollverlust}

Der Selfie-Modus führte aber noch zu einem anderen Effekt, der die Konstellation des Blickens zusätzlich verkomplizierte. Smartphones sind heute standardmäßig mit Frontkameras ausgestattet, die eine Aufnahme bei gleichzeitigem Blick auf das Display erlauben. Dementsprechend ist davon auszugehen, dass Abballa während des Livestreams sein eigenes Bild parallel betrachtete. Dies kann als eine Form der Selbstvergewisserung und Bildkontrolle gedeutet werden. Die "Märtyrer«-Inszenierung des Attentäters vollzog sich in einer kontinuierlichen Feedbackschleife zwischen dem Blick auf sich selbst und den Reaktionen seines Medienpublikums. Die für Selfies charakteristische Kontrolle über das eigene Bild kollidierte in diesem Fall jedoch mit der Unplanbarkeit, die mit jeder Liveübertragung einhergeht. Auch darin unterscheidet sich der Livestream des Attentäters von den meisten anderen Videotestamenten des IS, die vor der Veröffentlichung zahlreiche Nachbearbeitungsprozesse durchlaufen, bevor sie von den Medienstellen des IS verbreitet werden. Das live gepostete und unautorisierte Videotestament Abballas schien für den Islamischen Staat hingegen mit besonderen Risiken verbunden gewesen zu sein, so zeigt der nachträgliche Umgang damit. Obwohl sich der IS nur kurze Zeit später über seine Nachrichtenagentur Amaq offiziell zum Attentat in Magnanville bekannte, wurde das Video nur in stark gekürzter Version verbreitet. Der Teil, in dem der dreijährige Sohn der Ermordeten im Hintergrund auftauchte und in dem Abballa seine Unentschiedenheit im Hinblick auf dessen Schicksal zum Ausdruck brachte, war in der IS-Version nicht zu sehen (vgl. Rubin/Blaise 2016). Nach Angaben der New York Times entbrannte auf Twitter eine Debatte über die Gründe für diese Entscheidung. Wurde einerseits darüber spekuliert, ob die Bilder eines wehrlosen Kleinkindes selbst für die Standards des Islamischen Staats zu weit gingen, vermuteten andere, dass das Zögern des Attentäters, den Jungen ebenfalls zu ermorden, vom IS als Schwäche ausgelegt wurde (ebd.). Was auch immer den Ausschlag gegeben hatte: Für die Propagandamaschinerie des Islamischen Staats schien das Bild des verängstigten Jungen hinderlich gewesen zu sein. Die auf Anerkennung seiner »Brüder« angelegte Selbstdarstellung als »gnadenloser« Mörder schien durch die (anscheinend nicht antizipierte) Anwesenheit

Die Kategorie des Martyriums (arab. Istishād) spielt in Videotestamenten von Selbstmordattentätern des IS eine zentrale Rolle. Wie ich an anderer Stelle argumentiere, geht es bei den Selbstinszenierungen u.a. darum, schon zu Lebzeiten ein Bild als Märtyrer zu erschaffen und damit die »Belohnung « und Verehrung angesichts des geplanten Attentats vorwegzunehmen und für die Zukunft abzusichern (vgl. Straub 2021; 2019). 
des Dreijährigen irritiert worden zu sein. Unter den Bedingungen des situierten und vernetzten Livestreaming wird besonders deutlich, dass sich die Effekte eines invektiven Aktes den Intentionen der Invektierenden mitunter entziehen können.

\section{Soziale Netzwerke in der Kritik}

Es dauerte ganze 11 Stunden bis Facebook den Livestream zusammen mit dem Profil des Attentäters von der Plattform löschte. Zu diesem Zeitpunkt wurde das Video bereits mehrfach heruntergeladen, kopiert und auf anderen Kanälen weiterverbreitet. Auch wenn Facebook kurz nach dem Anschlag von Magnanville eine klare Stellung gegen das Posten terroristischer Gewaltbilder bezog und sein Bemühen beteuerte, Livestreams von Gewalttaten in akuten Fällen zu unterbrechen (vgl. Ehrenberg/Sagatz 2016), machte der Fall unmissverständlich deutlich, welche Schwierigkeiten das Unternehmen bei der Moderation dieser Art von Inhalten hat. Im Unterschied zu gewöhnlichen Facebook-Posts, deren Rezeption sich meist über einen längeren Zeitraum ausdehnt, ist das Zeitfenster, in dem ein Livestream wahrgenommen wird, wesentlich kleiner. Eine Prüfung der Inhalte hinkt zwangsläufig hinterher. Facebook ist mit diesem Problem nicht allein. Auch die zu Twitter gehörende Livestreaming-App Periscope und das zu Amazon gehörende LivestreamingPortal Twitch wurden zum Schauplatz von Gewalttaten, die sich scheinbar ungehindert verbreiten konnten. Allein im Jahr 2016 machten mehrere Fälle Schlagzeilen, die den öffentlichen Druck auf die Online-Anbieter erhöhten. ${ }^{7}$ Als Reaktion auf diese Entwicklungen schloss sich Facebook gemeinsam mit Twitter, YouTube und Microsoft im Juli 2017 zum Global Internet Forum to Counter Terrorism (GIFCT) zusammen, um dem Missbrauch der jeweiligen Plattformen durch terroristische und gewaltsame Akteure entgegenzuwirken. ${ }^{8}$ Dass Facebook die selbst formulierten Ziele auch zwei Jahre nach Gründung des GIFCT nicht annähernd erreicht hatte, wurde spätestens dann klar, als ein rechtsextremer Attentäter sein Massaker im neuseeländischen Christchurch per Livestream übertrug.

Im April 2016 wurde eine Frau angeklagt, die die Vergewaltigung ihrer Freundin auf Periscope übertrug; einen Monat später machte Periscope erneut negative Schlagzeilen, als eine Französin ihren Selbstmord in Echtzeit filmte. Auch Twitch wurde im Mai 2016 zum Schauplatz einer Vergewaltigung, die live per Audiostream zu verfolgen war. Im Januar 2017 stand schließlich auch Facebook Live wieder verstärkt in der Kritik, als ein Mann in Chicago vor laufender Kamera von vier Teenagern brutal misshandelt wurde.

8 Siehe die Webseite der GIFCT: https://www.gifct.org/about/ [Zuletzt eingesehen 14.7.2021]. 


\section{2) Das Attentat als Egoshooter-Spiel}

Das Massaker von Christchurch schien dem Töten im Livestream eine neue Dimension zu verleihen. Der aus Australien stammende Brenton Tarrant griff am 15.3.2019 zwei Moscheen in Christchurch mit Sturmgewehren an, tötete 51 Menschen und verletzte Dutzende weitere schwer. Mit einer Helmkamera filmte er seine Tat und stellte sie über Facebook Live ins Netz. Den Angaben von Facebook zufolge verfolgten rund 200 Menschen die 17-minütige Liveübertragung des Täters, die sich anschließend auf radikalen Imageboards wie 8chan, aber auch über Soziale Medien wie Twitter und YouTube millionenfach verbreitete (vgl. Brühl/Ernst 2019). Alles was über die Tat bekannt ist, weist darauf hin, dass der Anschlag explizit für seine Übertragung im Netz geplant war. Tarrant kündigte sein Attentat auf 8chan mit dem Verweis an, dass es Zeit sei das »Shitposting " $\mathrm{zu}$ beenden und einen echten Beitrag im realen Leben zu leisten (Sieber 2020:57). Damit bezog er sich auf die Subkultur sogenannter Imageboards, die mit ihren provokativen, teils ironischen Posts darauf abzielt, weniger internetaffine Öffentlichkeiten zu irritieren und Aufmerksamkeit innerhalb der eigenen Netzgemeinschaft zu erregen. Insbesondere die 8chan-Unterseite/pol/- Politically Incorrect, auf der auch Tarrants Post erschien, wurde als Inkubator für rechtsextreme, rassistische, antisemitische und frauenfeindliche Inhalte bekannt, die die Grenze zwischen Ironie und ernsthafter Gewalt zunehmend überschreiten. Neben seiner Ankündigung, einen Angriff gegen »Eindringlinge « durchzuführen, postete Tarrant dort auch ein 74-Seiten langes Manifest sowie den Link zu seinem Facebook Livestream, der knapp 20 Minuten später den tatsächlichen Anschlag übertrug.

\section{Der tötende Blick zwischen Spiel und Realität}

Die mordende Perspektive des Attentäters wurde zu einem grausamen Spektakel, das viele an die Ästhetik von Egoshooter-Spielen denken ließ. Der Gewehrlauf der Tatwaffe fällt hier mit einem invektiven Blickregime in eins, das das Morden als Spiel inszeniert und die realen Opfer damit verhöhnt und dehumanisiert. Schon Anders Breivik, der im Juli 2011 auf der norwegischen Insel Utøya 77 Menschen tötete und auf den sich Tarrant explizit als Vorbild bezog, wies enge Verbindungen zur Egoshooterszene im Internet auf. Sowohl in seinem Manifest als auch vor Gericht bezeichnete Breivik seine virtuellen Tötungserfahrungen als Training für sein reales Attentat, das er selbst wie ein Computerspiel plante. Wie Tarrant hatte auch Breivik vor, seine Tat per Video aufzunehmen und online zu verbreiten, auch wenn dies in seinem Fall letztlich scheiterte (vgl. Sieber 2020: 51).

Der Zusammenhang von Computerspielen und realer Gewalt wird seit dem Aufkommen von Egoshootern äußerst kontrovers diskutiert. Dabei steht immer wieder die Frage im Mittelpunkt, inwiefern Spiele, in denen Tötungshandlungen 
gegen Menschen simuliert werden, auch zu einer erhöhten Aggressivität im realen Leben führen und mitunter eine Mitschuld an Gewaltkriminalität tragen. ${ }^{9}$ Auch wenn das Spielen von Egoshootern nicht automatisch zu einer höheren Gewaltbereitschaft im realen Leben führt, machen die rechtsextremen Attentate der vergangenen Jahre deutlich, dass das Ineinandergreifen von Online-Radikalisierung und Gamerszene zu einem »neuen Tätertypus« (Baeck/Speit 2020: 8) geführt hat: Rechtsterrorist:innen, deren Hass sich im Umfeld einer zynischen Internetsubkultur herausbildet, die ihre Taten im Netz ankündigen, in der Logik von Computerspielen planen und schließlich per Livestream ins Netz stellen. In den Monaten nach dem Massaker in Christchurch folgten vier weitere rechtsextreme Attentäter, die diesem neuen Tätertypus entsprachen. ${ }^{10}$ Schon die von den Attentätern verfassten und online verbreiteten Beschreibungen der geplanten Tat lesen sich häufig wie Spieleanleitungen mit Zielen und »Achievements«, die es zu erreichen gelte (Sieber 2020: 48). Die »Gamifizierung« rechter Attentate zeigt sich aber auch an sogenannten »Highscore«-Tabellen, die im Internet kursieren, und Attentätern nicht nur für die Anzahl ihrer Todesopfer, sondern auch für das Livestreaming ihrer Tat eine höhere Punktezahl vergeben (vgl. ebd.: 50). Die mediale Vermittlung des Anschlags scheint zum eigentlichen Ziel des Mordens zu werden. Das als Egoshooter inszenierte Massaker bedient die exzessive Lust der Shitposter, immer noch radikalere Inhalte zu verbreiten, die keinen Unterschied mehr machen zwischen Satire und Ernst, zwischen Spiel und Realität. Auch hier erfüllt der live übertragene invective gaze des Attentäters also eine doppelte kommunikative Funktion. Die größtmögliche Demütigung der Todesopfer - und mit ihnen aller Angehörigen und Mitfühlenden - zielt zugleich auf eine größtmögliche Aufmerksamkeit und Anerkennung innerhalb einer Netzgemeinschaft von Gleichgesinnten ab. Tatsächlich erzeugte der Livestream im rechtsextremen Online-Milieu eine enorme Resonanz. Sowohl auf Facebook als auch auf 8chan »jubelten« seine Anhänger angeblich, während sie die Morde live verfolgten (Sieber 2019: 59). Allein die Tatsache, dass das Video erst 12 Minuten nach Ende des Livestreams von Facebook-User:innen als beanstandungswürdig gemeldet wurde, verweist darauf, dass das Live-Publikum vorwiegend aus Mitgliedern der radikalen Netz-Community bestand, die dem Attentat zumindest billigend gegenüberstanden. Vom medialen >Erfolg〈des Livestreams

Einen guten Überblick über die Forschungslage zum Thema Cewalt und Computerspiel bietet Venus 2018.

10 Darunter der Angriff auf eine Synagoge am 27.4.2019 in Poway (USA), das Attentat auf vermeintliche mexikanische Einwanderer am 3.8.2019 in El Paso (USA), der Anschlag auf eine Moschee am 10.8.2019 in Baerum (Norwegen) und der am 9.10.2019 verübte Anschlag auf eine Synagoge in Halle. Dass es sich dabei ausschließlich um männliche Täter handelte, mag in diesem Kontext kaum verwundern. So wurde vermehrt auf die frauenfeindliche Gesinnung rechtsextremer Internet-Subkulturen hingewiesen, die häufig mit der ebenfalls männlich dominierten Gamerszene in Verbindung steht, vgl. Nagle 2017. 
zeugen schließlich auch die zahlreichen Kopien, die nach wie vor in den dunkelsten Ecken des Netzes kursieren, sowie die Memes, die auf 8chan gepostet wurden und Brenton Tarrant als Heiligen glorifizieren (vgl. Tarabay 2019). Schließlich verbreitete sich sogar ein von User:innen erstelltes Egoshooter-Spiel im Internet, das die Gamifizierung des Attentats auf eine weitere Ebene hob und die mordende Perspektive im Sinne eines zynischen Reenactments nachspielbar machte (vgl. Sefa 2019).

\section{Immersion in das Töten}

Die Inszenierung der Tat als Egoshooter-Spiel wirft unweigerlich die Frage auf, in welchem Verhältnis der invective gaze des Täters und dem invective gaze des Medienpublikums steht. Wie schon beim ersten Beispiel setzte die Liveschaltung auf eine Involvierung des Medienpublikums als Zeug:innen. Diese bestand jedoch weniger im Angebot einer direkten Kontaktaufnahme mit dem Täter (obwohl auch hier live kommentiert werden konnte), sondern im Angebot einer Immersion in das Töten selbst. Statt mit dem Attentäter >Auge in Auge in Kontakt zu treten, wie dies bei Abballas Selfie-Video der Fall war, waren die Zuschauenden gezwungen, den Blick des Mörders selbst nachzuvollziehen. Auch ohne den Livestream gesehen zu haben, kann man sich nur zu gut vorstellen wie sich jede Kopfbewegung, jedes Beben während eines abgefeuerten Schusses, vielleicht sogar das Atmen des Täters audiovisuell an die Betrachtenden vermittelt haben muss. Das Authentizitätsversprechen des Livestreaming scheint sich hier ins Extrem zu wenden und geradezu pornografische Züge anzunehmen. ${ }^{11}$ Wie bei Egoshooter-Spielen ragte das Sturmgewehr des Täters immer wieder ins Blickfeld der Kamera (Baeck/Speit 2020: 9). Die perspektivische Gestaltung wies den Zuschauenden im wahrsten Sinne einen >Platz im filmischen Raum zu und bezog sie auf körperlich erfahrbare Weise in das Geschehen mit ein. Die »Erfahrung einer gefühlten Präsenz in künstlichen oder digital erzeugten Räumen « wird aus film- und medienwissenschaftlicher Sicht gemeinhin als »immersiv« bezeichnet (Curtis 2008: 90). Wenngleich Immersion durchaus ein »intellektuell stimulierender Prozess« sein kann, beschreibt der Kunsthistoriker Oliver Grau die virtuelle Immersionserfahrung als einen mentalen Zustand "gesteigerter emotionaler Involvierung «, die in den meisten Fällen mit einer "verminderten kritischen Distanzfähigkeit« zum Dargestellten einhergehe (Grau 2003: 13). Der ästhetische Anschluss an das Blickregime des Egoshooters verknüpft den Livestream des Attentäters mit denselben affektiven Registern, die auch in interak-

11 Von »Attentatspornografie«spricht etwa auch der Medienwissenschaftler Bernhard Pörksen in Bezug auf die Berichterstattung über den Livestream des Attentats in Christchurch (Pörksen 2019). 
tiven Computerspielen aufgerufen werden und mit (zumeist positiven) Erfahrungen von Eigenwirksamkeit, Wettbewerb, Flow oder Immersion verbunden sind.

Diese »emotionale Involvierung « wird zusätzlich gesteigert, wenn das Betrachtete eine live bezeugte Realität darstellt. Mit der Liveschaltung schloss Tarrant an eine in der Gaming-Community gängige Praxis an, Spielsequenzen in Echtzeit auf YouTube oder auf Gaming-Plattformen wie Twitch zu streamen, während parallel die eigenen Reaktionen auf das Spielgeschehen als Bild-im-Bild angezeigt werden. Was von den Abonnent:innen solcher Streams konsumiert wird, ist weniger das Spiel an sich, sondern die Affiziertheit der Spielenden, die sich über die mimischen, gestischen und verbalen Reaktionen vermittelt. ${ }^{12}$ Ähnliches lässt sich auch über den Livestream von Brenton Tarrant sagen. Das zutiefst Verstörende an diesem Video ist nicht nur der Umstand, dass grausame Morde ins Blickfeld einer Kamera treten, sondern auch die Immersion in die Intensität seiner Erregung. Der mit dem Anvisieren und Töten in eins fallende invective gaze des Täters überträgt sich auf geradezu körperlich-affektive Art und Weise an die Betrachtenden, die damit - im Unterschied zur sprachlichen und symbolischen Interaktion während des Livestreams aus Magnanville - noch einmal ganz anders an das medial bezeugte Geschehen gebunden werden.

\section{Ethik des Zusehens}

Wenn das Töten nicht nur vor der Kamera, sondern für die Kamera stattfindet, wenn die mediale Liveübertragung also zum eigentlichen Ziel des Mordens wird, rückt die Frage nach einer Mittäterschaft des Medienpublikums mit neuer Brisanz in den Vordergrund. Mit ihrem »Christchurch Call« zog die neuseeländische Premierministerin Jacinda Ardern Online-Konzerne wie Facebook zur Rechenschaft. Soziale Medien seien »der Herausgeber und nicht nur der Briefträger« solcher Gewaltbilder, so Ardern (Brühl/Ernst 2019). Sie schließt damit an die in der Terrorforschung weit verbreitete These an, dass Medien der Berichterstattung eine Mitschuld am >Erfolg< der Attentate haben, die in der medialen Aufmerksamkeit ihr eigentliches Ziel sehen (vgl. Allison/Cook 2007: 87f.). Dutzende Regierungen und große Internetkonzerne, darunter Facebook, Twitter, YouTube und Microsoft folgten dem Aufruf der neuseeländischen Regierungschefin und verpflichteten sich da$\mathrm{zu}, \mathrm{Maßnahmen}$ gegen die Verbreitung gewalttätiger Inhalte zu ergreifen (»Christchurch Call« 2019). Sind die Bilder jedoch einmal in der Welt, lassen sie sich nur

12 Dies trifft im Wesentlichen das, was Michael Richardson und Kerstin Schankweiler mit ihrem Konzept des »Affective Witnessing« beschreiben. Gemeint ist ein bestimmter Modus der relationalen Zeugenschaft, in dem das, was bezeugt wird, das Affizierungsgeschehen selbst ist (Richardson/Schankweiler 2018). 
noch schwer kontrollieren. Das machen nicht zuletzt die zahlreichen Kopien des Christchurch-Videos deutlich, die bis heute im Netz kursieren.

Neben der Kritik an den Sozialen Netzwerken und klassischen Medien wurde daher vermehrt auch an die Verantwortung der einzelnen User:innen appelliert und darüber diskutiert, inwiefern allein das Ansehen und Teilen eines solchen Videos strafbar ist (vgl. Tarabay 2019). Die Frage nach einer Mittäterschaft durch Zusehen wurde schon am Beispiel der Enthauptungsvideos virulent, die ab 2002 von al-Qaida und IS online verbreitet wurden. Der Kunsthistoriker Horst Bredekamp hat argumentiert, dass die Videoaufnahme in diesen Fällen den eigentlichen Anlass für das Töten liefert (vgl. Bredekamp 2016: 23). Aus diesem Grund, so schreibt auch die Kunsthistorikerin Charlotte Klonk ist »jeder Klick auf den entsprechenden Internetseiten, jede Betrachtung der Aufnahmen ein Ansporn zu weiteren Taten.« Enthauptungsvideos, so schreibt Klonk, »zwingen zur unfreiwilligen Komplizenschaft und haben Mord als Konsequenz (Klonk 2017: 135). Anders gesagt: Der Blick auf diese Bilder ist mit dem Gewaltakt selbst verknüpft. Dies gilt umso mehr für die Liveübertragung des Massakers in Christchurch. Hier sind die durch Klicks vermittelten Blicke des Medienpublikums nicht nur Ansporn zu weiteren Taten, sie wurden zu Bestätigungen und Amplifikationen der Tat im Vollzug. Ob das LivePublikum im Einzelnen der Tat unterstützend oder ablehnend gegenüberstand, scheint dabei kaum ins Gewicht zu fallen, da allein die Erhöhung der Klickzahl den Zielen des Attentäters in die Hände spielte. Es gibt also gute Gründe dafür, diese Bilder nicht aufzusuchen, anzusehen oder gar weiterzuverbreiten. Gleichzeitig ist eine kritische Rahmung und Kontextualisierung aus bildwissenschaftlicher Perspektive dringend angezeigt. Umso mehr, wenn es sich um ein Massaker handelt, das aufs Engste mit den Bildpraktiken und invektiven Blickregimes heutiger Online-Kulturen verbunden ist.

\section{3) Livezeugnis aus Opferperspektive}

Als drittes Beispiel soll schließlich ein Handyvideo diskutiert werden, das anders als die bisher diskutierten Fällen nicht aus Sicht des Täters, sondern aus Sicht des Opfers gefilmt ist. Es zeigt den Tod des Schwarzen US-Bürgers Philando Castile, der am 6.7.2016 während einer Polizeikontrolle in Saint Paul, Minnesota, vor den Augen seiner Freundin und deren vierjähriger Tochter in seinem Auto erschossen wurde. Nachdem das Auto wegen eines defekten Rücklichts angehalten wurde, informierte Castile den Polizisten, dass er eine Waffe samt Waffenbescheinigung bei sich trage. Obwohl Castile mehrmals beteuerte, diese nicht zu ziehen, dauerte es nur wenige Sekunden bis der Polizist Jeronimo Yanez sieben Schüsse auf den 32Jährigen abfeuerte. Erst zehn Minuten später wurde Castile erste Hilfe geleistet, zwanzig Minuten später starb er in der Notaufnahme. 
Kurz nachdem Philando Castile erschossen wurde, startete dessen Freundin Diamond Reynolds vom Beifahrersitz aus mit ihrem Smartphone einen Livestream auf Facebook Live. Mit erstaunlicher Klarheit und bemerkenswerter Fassung schilderte sie für »alle, die zusehen« den genauen Tathergang und dokumentierte die unmittelbaren Folgen der Tat bis hin zu ihrer Abführung auf dem Rücksitz eines Polizeiwagens. ${ }^{13}$ Das rund 10 Minuten lange Video offenbart ein komplexes BlickVerhältnis zwischen Opfer, Täter und Medienpublikum: Reynolds richtete die Kamera abwechselnd auf sich selbst, auf ihren sterbenden Freund, sowie auf die Polizeibeamten, die ihrerseits mit Waffen die Filmende ins Visier nahmen und auf sie zielten. Über weite Strecken hinweg ließ der Livestream die Facebook-User:innen mit den Augen des (überlebenden) Opfers sehen. Die oben beschriebene Immersion in das Töten fand hier unter umgekehrten Vorzeichen statt: Vermittelt durch die Handykamera richtete sich die Waffe, mit der Yanez an Castiles blutendem Körper vorbei auf die Filmende zielte, gleichzeitig auf alle Betrachtenden (Abb. 2). Jede/r einzelne, der/die das Video rezipierte, musste die tödliche Bedrohung am eigenen Körper nachempfinden. Vermittelt durch die Kamera des Opfers wurde das Medienpublikum vom Übergriff und der Gewalt der Polizisten ebenso beschämt und erniedrigt. Die verkörperte Kameraperspektive versetzte die LiveFollower:innen wenige Augenblicke später erneut in die entwürdigende Position Reynolds', als diese aufgefordert wurde, das Auto zu verlassen und auf die bewaffneten Polizisten zuzugehen, während sie, zusammen mit den Medienzeugen auf Facebook, zusehen musste wie ihre vierjährige Tochter auf der anderen Seite von einem Polizisten festgehalten wurde (Abb. 3). Durch den ständigen Wechsel in den Selfie-Modus änderte sich die Perspektive der Machtlosigkeit jedoch immer wieder in einen Modus der Selbstermächtigung. Ihre Facebook Follower:innen wurden damit nicht nur zu unmittelbaren Augenzeugen, sondern auch zu Medienzeugen zweiter Ordnung, die Reynolds' subjektives Zeugnis anhörten und ihren Kommentar der Ereignisse live verfolgten (Abb. 4). Selbst als sie von den Polizeibeamten festgenommen wurde und ihr Telefon Minutenlang auf dem Boden lag und nur einen schwarzen Bildschirm zeigte, adressierte sie ihr Medienpublikum explizit (»They threw my phone, Facebook«). Später mobilisierte sie ihre Freunde, zum Ort des Geschehens zu kommen. Einige ihrer Follower:innen reagierten wiederum unmittelbar auf ihr Zeugnis mit Kommentaren und Handlungsaufforderungen wie "Don't stop recording« (Stelter 2016). Ihr Livestream ist damit ein eindrückliches Beispiel für die Relationalität und Konnektivität von Zeugenschaft, die in Zeiten Sozialer Medien »als performative Interaktion von Körpern und Blicken« operiert (Papailias 2019:118). 
Abb. 2-4
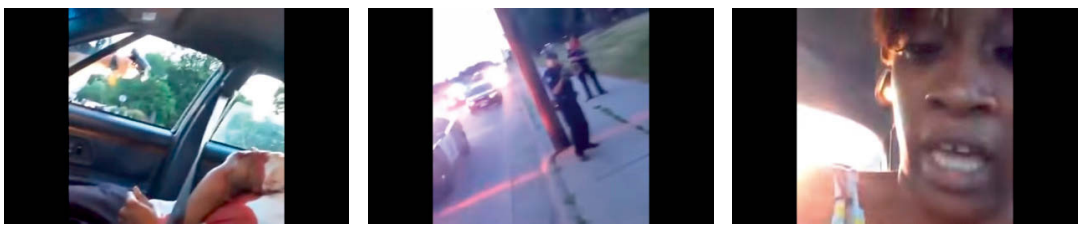

\section{Das Videozeugnis als Anklage und Public Shaming}

Im Unterschied zu den oben diskutierten Tätervideos, in denen das Blicken teilweise mit dem Töten in eins fällt, ist die Frage nach dem invektiven Potenzial dieser interagierenden Blicke weitaus komplexer. Die tödliche Handlung des Polizisten ist selbst als Resultat eines (weißen) rassistischen Blickregimes zu begreifen, das sich über Jahrhunderte in die Geschichte der USA eingeschrieben hat und die Vorstellung des afroamerikanischen Mannes als Aggressor normalisiert hat (vgl. Smith 2000). Wie zahlreiche Untersuchungen zeigen, wird die kulturelle Imagination einer »black criminality « auch heute noch zur Rechtfertigung von Polizeigewalt instrumentalisiert (vgl. Weissman 2019; Fain 2016). Auch der Polizist Yanez sagte später vor Gericht aus, er habe »Angst um sein Leben" gehabt, weil er glaubte, dass Castile nach seiner Waffe statt nach seinem Ausweis griff (vgl. Haas 2017). Reynolds' Darstellung kontert dieses rassistische Blickregime, indem sie die Unschuld ihres sterbenden Freundes bezeugt und damit der allgegenwärtigen Kriminalisierung von Schwarzen Widerstand leistet. Der strukturelle invective gaze der Polizisten wird mit einem Gegen-Blick gekontert, der die Gewalt dieses Blickregimes offenlegt und anklagt.

Reynolds verzichtete dabei auf direkte verbale Angriffe oder Beleidigungen der Polizisten. Ganz im Gegenteil: Es ist geradezu schockierend, dass sie den verantwortlichen Polizisten selbst dann noch höflich als »Sir« adressierte, nachdem dieser ihren Freund kaltblütig erschossen hatte (»You shot four bullets into him, Sir.«). Die Anklage, die sich durch ihren Livestream vermittelt, operiert auf einer viel fundamentaleren Ebene. Allein die Tatsache, dass die unter Schock stehende Frau ihre Handykamera gegen die Waffe - die lebensbedrohliche invektive Geste - des Polizisten wandte, kann als Akt der Ermächtigung und Gegenwehr interpretiert werden. Wenngleich der Vergleich zwischen Kamera und Waffe, zwischen Fotografieren und Schießen, in einer langen theoretischen und aktivistischen Tradition steht, ${ }^{14}$ führte die Allgegenwärtigkeit von Handykameras in aktuellen Pro-

14 Susan Sontag hat auf die aggressive und besitzergreifende Dimension der Fotografie sowie auf die sprachlichen Parallelen zwischen Fotografieren und Schießen hingewiesen (vgl. Son- 
testkulturen dazu, dass das Bildermachen als Mittel der politischen Agitation neue Aufmerksamkeit erfuhr (vgl. Schankweiler 2019; Schankweiler/Straub/Wendl 2019). Reynolds' Livestream ist Ausdruck einer Kultur von selbstbewusst auftretenden »citizen camera-witnesses«, die sich mit ihren Körpern in die Schusslinie begeben, um staatliche Gewalt zu dokumentieren und dieser ein eigenes Blick-Regime >von unten entgegenzusetzen (vgl. Andén-Papadopoulos 2013). Ziel ist es, ebenso beweiskräftige wie affektive Bilder zu schaffen, die in der Lage sind, Wahrheiten ans Licht zu bringen und Menschen zu mobilisieren. Der Glaube an die aufklärerische Wirkmacht und Evidenz solcher Bilder prägt auch Reynolds' Livestream. Besonders deutlich wird dies gegen Ende der Aufnahme, als sich die Frau zusammen mit ihrer Tochter auf dem Rücksitz eines Polizeiwagens befand, während das Mobiltelefon vor ihr auf dem Schoß lag. Obwohl ihre Hände zu diesem Zeitpunkt gefesselt waren, schaffte sie es, die Kamera immer wieder auf die Polizisten außerhalb des Autos zu richten und den Verantwortlichen zu identifizieren: »That's the police officer over there that did it with the black on.« In einem späteren Interview bestärkte Reynolds ihr Anliegen, mit dem Livestream ein Zeugnis zu schaffen, das von der Polizei nicht manipuliert werden könne und alle Menschen in die Position versetze, "mit eigenen Augen « zu sehen, was wirklich passiert ist: "I wanted everyone in the world to know that no matter how much the police tamper with evidence, [...] no matter how they manipulate our minds to believe what they want, I wanted to put it on Facebook and go viral so that the people could see." (Mott 2016). Indem Reynolds die Handlungen der Polizisten publik machte, schuf sie ein Bildzeugnis, das all diejenigen beschämt, die rassistisch motivierte Polizeigewalt zu verharmlosen oder zu vertuschen versuchen (vgl. Fain 2016). Ohne selbst verbal zu beschämen, kann Reynolds' Livestream als Mittel des öffentlichen Shaming gedeutet werden, dessen invektive Kraft gerade in seinem Anspruch auf Authentizität und Evidenz begründet liegt.

Das Format des Facebook Livestreams spielte dafür eine entscheidende Rolle. Die Simultaneität von bezeugtem Ereignis und medialer Übertragung macht technische Nachbearbeitungsprozesse wie Schnitte, Montagen oder Spezialeffekte weitaus schwieriger (wenn auch nicht gänzlich unmöglich). Mehr noch als andere mobile Videozeugnisse sind Livestreams mit dem Versprechen einer unmanipulierbaren Aufzeichnung der Realität verbunden. Dieser Anspruch liegt auch Reynolds' Aufnahme zugrunde, wenn sie ihr Video explizit den »manipulierten Zeugnissen« der Polizei gegenüberstellt. Hinzu kommt die weitgehend ungefilterte (und schwer filterbare) Verbreitung von Livestreams in den Sozialen Medien. Wie oben

tag 2001: 14). Die Symbolik der Kamera als Waffe wurde immer wieder auch von aktivistischen Filmemacher:innen weltweit aufgerufen, die ihre filmische Arbeit als Teil des revolutionären Kampfes verstanden (vgl. z.B. Solanas und Getino 1970). 
bereits deutlich wurde, sind Inhalte in Echtzeit weitaus schwerer zu prüfen, zu löschen oder zu moderieren als gewöhnliche Posts. Im Fall der Tätervideos wurde dies Facebook zum Verhängnis. Indem sich die Livestream-Funktion (weitgehend) der Kontrolle der Plattform-Anbieter entzieht, birgt sie zugleich aber auch das Potenzial, Aufnahmen publik zu machen, die ansonsten der Zensur der Filter unterliegen würden, so argumentiert der Medienwissenschaftler Benjamin Burroughs (vgl. Dewey 2016). Einmal in der Welt, lassen sich die Bilder nicht mehr ungesehen machen. Auch in Reynolds' Fall scheiterte der Versuch, die Verbreitung des Videos zu unterbinden. Nur wenige Stunden nach der Liveübertragung wurde der Post ohne weitere Erklärung von Reynolds' Facebook-Profil entfernt. Der offiziellen Stellungnahme von Facebook zufolge handelte es sich um einen »technischen Fehler«, für den sich das Unternehmen entschuldigte, für den es zugleich aber mit Vorwürfen der Zensur konfrontiert wurde (vgl. Stelter 2016). Das Video war rund eine Stunde später wieder verfügbar, nun aber mit einem Warnhinweis für besonders drastische Inhalte. Die Gründe für das temporäre Verschwinden sind umstritten: Womöglich wurde das Video von User:innen als beanstandungswürdig gemeldet, daraufhin von Facebooks Algorithmen erfasst und von Moderator:innen gelöscht, wie dies standardmäßig bei Facebook gehandhabt wird. Nach Aussage von Reynolds wurde die Löschung hingegen von der verantwortlichen Polizeibehörde selbst veranlasst, die das Mobiltelefon kurz nach ihrer Festnahme konfiszierte und sich Zugriff auf ihr Facebook-Profil verschafft haben soll (vgl. Thompson 2016). Wer auch immer die Entfernung des Posts zu verantworten hatte: Die virale Verbreitung des Videos, die schon während der Liveübertragung einsetzte, konnte dadurch nicht aufgehalten werden. Zum Zeitpunkt der Löschung lag die Klickzahl schon bei 1,5 Millionen. Nur drei Stunden nach der Liveschaltung hatte Reynolds' erschütterndes Zeugnis erste Proteste am Ort des Geschehens und vor der verantwortlichen Polizeizentrale in Saint Paul mobilisiert und trug dazu bei, dass der Fall innerhalb kürzester Zeit international bekannt wurde.

\section{Anklage im Livestream vs. Urteil vor Gericht}

Am 16.6.2017 wurde der verantwortliche Polizist Jeronimo Yanez von allen Anklagepunkten freigesprochen. Das Urteil war ein bitterer Schlag für die Angehörigen des Ermordeten und für alle, die für die Ziele der Black Lives Matter-Bewegung kämpften. Der Freispruch zeigte auch die Widersprüchlichkeit auf, die zwischen dem viralen >Erfolg ‘ des Videos und dessen juristischer Effektivität liegt. Trotz des Anspruchs, ein authentisches, unmittelbares und unmanipulierbares Zeugnis zu schaffen, spielte der Livestream vor Gericht als Beweismittel keine Rolle. Gerade im Zusammenhang mit Zeugnissen von Polizeigewalt wurde immer wieder deutlich, dass die Bilder je noch Kontext umgedeutet werden können und teilweise sogar als Beweise für die Unschuld der verantwortlichen Polizisten dienten. Dasselbe Video, 
das das brutale Vorgehen der Polizei gegen den Schwarzen US-Amerikaner Rodney King am 3.3.1991 bezeugte, wurde etwa auch von den Verteidigern der LAPDPolizisten instrumentalisiert, um deren Unschuld zu untermauern. Von den Verteidigern wurden jedoch nur Standbilder aus dem Video genutzt, die Kings Bewegungen auf eine Weise verzerrten, dass sie als Beweise für dessen potenzielle »Aggressivität« und »Animalität« herangezogen werden konnten (Weissman 2019: 7). Die invektive Stoßrichtung des Videos kehrte sich hier in ihr Gegenteil um: Statt die dargestellte, eigentlich unbestreitbare Polizeigewalt anzuklagen, wurde das Rodney King-Video genutzt, um die »weiße Paranoia« einer Bedrohung durch kriminelle Schwarze zu schüren und die weißen Polizisten als die eigentlichen Opfer darzustellen (Butler 1993: 19). Wie oben bereits angedeutet, wurde diese Imagination auch im Gerichtsprozess gegen Jeronimo Yanez instrumentalisiert. ${ }^{15}$ Angesichts der juristischen Ineffektivität vieler Videozeugnisse von Polizeigewalt war von einem Scheitern der »Repräsentation« die Rede: Und zwar in einem doppelten Sinne eines Scheiterns der Evidenzerzeugung und der politischen Repräsentation (vgl. Joselit 2015). ${ }^{16}$ Dennoch wäre es verfehlt, den Videos jegliche politische Wirkmacht abzusprechen. Wie Terry Weissman argumentiert, haben die Bildzeugnisse durchaus einen kumulativen Effekt: Sie stehen nicht allein, sondern lösen die Produktion weiterer Bilder aus, die gemeinsam ihren Widerstand artikulieren, in ihrer Gesamtheit einen politischen Handlungsdruck erzeugen und Machtgefüge verändern (vgl. Weissman 2019: 8). Der jüngste Fall des am 25.5.2020 durch die Polizei getöteten Schwarzen Amerikaners George Floyd verdeutlicht einerseits, wie wenig sich seit den vier Jahren nach Castiles Tod geändert hat und wie sehr die Realität der US-Amerikanischen Gesellschaft und speziell das Polizeisystem nach wie vor durch strukturellen Rassismus geprägt sind. Die politischen Reaktionen und Reformen, die auf die Veröffentlichung des Videos und die weltweiten Proteste folgten, stimmen zugleich (vorsichtig) optimistisch, dass der von Weissman beschriebene kumulative Effekt dieser Aufnahmen - und damit die Masse der beschämenden Blicke - auch zu einem politischen Wandel führen kann.

\section{»Are you sure you want to see this?«}

Nachdem das Video auf Reynolds' Facebook-Profil wiederhergestellt worden war, erschien es mit dem von Facebook standardmäßig eingeblendeten Warnhinweis: »Warning - Graphic Video. Videos that contain graphic content can shock, offend

\footnotetext{
15 Dies war auch in anderen Gerichtsprozessen gegen rassistisch motivierte Polizeigewalt zu beobachten, die sich in den Jahren vor Castiles Tod ereigneten (vgl. Weissman 2019: 7).

16 David Joselit bezog sich dabei insbesondere auf die Freisprechung des Polizisten, der den Schwarzen US-Amerikaners Eric Garner am 17.7.2014 tötete und dessen Tat durch zwei Videos an die Öffentlichkeit gelangte, die über YouTube verbreitet wurden.
} 
and upset. Are you sure you want to see this? « Die ethische Frage, die mit dem Ansehen dieses Videozeugnisses verbunden ist, wurde im akademischen Kontext durchaus kontrovers diskutiert - wenn auch unter ganz anderen Vorzeichen als im Fall der Tätervideos. Im Anschluss an Susan Sontag wurde einerseits argumentiert, dass die virale Verbreitung von Zeugnissen rassistisch motivierter Polizeigewalt $\mathrm{zu}$ einem gegenteiligen Effekt führen könne, nämlich zu einer zunehmenden Abstumpfung und Gleichgültigkeit gegenüber der Gewalt, die Schwarze tagtäglich erleiden (vgl. Juhasz 2016; Sontag 2003). Andererseits wurden Stimmen laut, die an unsere Verantwortung appellierten, sich diesen Bildern immer wieder aufs Neue auszusetzen: "Ignoring the specific videos of Castile and Alton Sterling's deaths is akin to them dying in vain, denying them postmortem acknowledgement as human beings«, schreibt die Anwältin und Historikerin Kimberly Fain (Fain 2016). Gerade weil Reynolds' Video vor Gericht ignoriert wurde, erscheint der Blick auf ihre Sichtweise und Darstellung der Ereignisse umso wichtiger. Ihr Livestream erhebt nicht nur Anklage gegen die physische Gewalt der dargestellten Polizisten, sondern auch gegen einen tief verwurzelten, strukturellen rassistischen invective gaze. Das Video anzuklicken und anzusehen heißt daher auch, diesem Gegen-Blickregime Gewicht zu verleihen. Hier zeigt sich die zutiefst ambivalente Ethik, die mit der medialen Zeugenschaft des Tötens im Livestream verbunden ist: Während uns das Ansehen des live übertragenen Massakers in Christchurch zur Mittäterschaft verpflichtet und Gewalt amplifiziert, kann eine größere Sichtbarkeit von Zeugnissen von Polizeigewalt dazu führen, diese Gewalt zu beschämen und bloßzustellen.

\section{Schluss}

Der Fokus auf die invektiven Blickdynamiken zwischen Tätern, Opfern und Medienpublikum ermöglicht einen neuen Zugriff auf das Social Media-Phänomen Livestreaming, das heute zu einer wirksamen Waffe von Terrorist:innen wie Aktivist:innen geworden ist. Am deutlichsten wird dies, wenn Tötungshandlungen zum Gegenstand der Darstellung werden (selbst wenn das eigentliche Morden unsichtbar bleibt). Gerade der Vergleich gegenläufiger Perspektiven zeigt, dass der invective gaze im Livestream sowohl zerstörerische als auch ermächtigende Potenziale umfassen kann. Wurde Facebook Live im Fall der Tätervideos zum Forum, vielleicht sogar zum »Herausgeber« der Gewalt, diente dieselbe Plattform im Fall der Tötung von Philando Castile dazu, die dargestellte Gewalt zu kontern.

Die Gegenüberstellung zwischen drei so unterschiedlichen Darstellungsformen des Tötens im Livestream zeigt auch, wie eng das Livestreaming mit anderen Bildgenres und -praktiken verknüpft ist, die sich im Kontext der interaktiven Online-Kultur des Web 2.0 herausgebildet haben. Das Selfie, das Egoshooter-Spiel und die konnektive Zeugenschaft sind jeweils durch spezifische Blickdynamiken 
geprägt, die wiederum ein spezifisches invektives Potenzial bergen. Während das Täter-Selfie die Ermordeten durch die Form der Situierung und Selbstdarstellung erneut entwürdigt, fallen beim live übertragenen Massaker Blicken, Filmen und Töten in eins. Im Unterschied zum Selfie nimmt die Egoshooter-Perspektive Bezug auf ein digitales Blickregime, das konstitutiv mit der exzessiven Lust an der Herabsetzung und Tötung verbunden ist und im Fall des Christchurch-Attentats von der virtuellen in die reale Welt übertragen wurde. Das dritte Beispiel knüpft hingegen an die Zeugenschafts-Praxis von »citizen camera witnesses« an, die mit dem Versprechen von Authentizität und Evidenz einhergeht und deren invektive Wirkmacht in einer Entlarvung und Bloßstellung liegt. Gerade beim letzten Fall wird aber auch deutlich, wie schnell sich die invektive Stoßrichtung eines solchen Videos je nach Kontextualisierung und Rahmung (etwa vor Gericht) verändern kann.

Die Wahl des Livestreams spielt für die Beschämungs- und Herabsetzungsdynamiken in allen drei Fällen eine zentrale Rolle. Entscheidend ist hier vor allem das Verhältnis zwischen den jeweiligen Sendenden der Aufnahme und den Interagierenden vor den Bildschirmen, die das Geschehen in Echtzeit mitverfolgen, teilen oder kommentieren können und damit auf bisher nicht dagewesene Art beteiligt sind. Aus diesem Grund liegt es nahe, von einem >neuen invective gaze im Livestream zu sprechen. Die Involvierung der User:innen geschieht einerseits auf affektiver Ebene, etwa durch die Immersion in die Ich-Perspektive von Täter oder Opfer, andererseits aber auch auf der Ebene der Handlung: Sowohl der Attentäter von Magnanville als auch Diamond Reynolds adressierten ihre FacebookFollower:innen mit expliziten Ansprachen und Aufforderungen. Durch den direkten Blick in die Kamera war es dem Medienpublikum möglich, mit den Filmenden live in Kontakt zu treten und die Situation durch im Bild sichtbare Likes oder Dislikes, durch Emoticons, Kommentare oder Handlungsaufforderungen ihrerseits zu beeinflussen (»Don't stop recording«). Dies ist auch dann der Fall, wenn die Möglichkeit zur Face-to-Face-Kommunikation mit dem Sender ausbleibt, wie im Fall des Christchurch-Attentats. Wenn die Übertragung im Livestream, wie hier, zum eigentlichen Anlass des Mordens wird, ist ein unschuldiges Ansehen und Anklicken nicht mehr möglich. Wird der Tatort auf diese Weise in den digitalen Raum erweitert, rückt allerdings nicht nur die ethische Verantwortung der einzelnen User:innen in den Vordergrund, sondern auch (und gerade) die Verantwortung der jeweiligen Plattformen mit ihren strukturellen Ermöglichungszusammenhängen. Der spezifische invective gaze, der sich unter den Bedingungen des Livestreaming realisiert, zeichnet sich gerade durch diese komplexen - medial bedingten, affektiven und ethischen - Verflechtungen von Blicken aus, die sowohl innerhalb als auch außerhalb des Bildfelds wirksam werden und den netzbasierten invective gaze damit als grundlegend relationales Geschehen charakterisieren. 


\section{Literaturverzeichnis}

Allen, Peter/Summers, Chris/Robinson, Julian (2016): »Pictured: Islamist Posts Facebook Video Saying He Slaughtered Couple. Dailymail Online«. https://w ww.dailymail.co.uk/news/article-3639878/Policeman-stabbed-death-outside -Paris-home-attacker-holding-officer-s-wife-son-hostage.html. (13.06.2016) [letzter Zugriff 14.7.2021]

Allison, Olivia/Cook, David (2007): Understanding and Addressing Suicide Attacks: The Faith and Politics of Martyrdom Operations. Westport: Praeger Security International.

Andén-Papadopoulos, Kari (2013): „Citizen Camera-Witnessing: Embodied Political Dissent in the Age of >Mediated Mass Self-Communication«. In: New Media Society, Jg. 16, Nr. 5, S. 753-769. DOI: 10.1177/1461444813489863.

Baeck, Jean-Philipp/Speit, Andreas (2020): »Von der virtuellen Hetze zum Livestream-Attentat«. In: Baeck, Jean-Philipp/Speit, Andreas (Hgg.): Rechte Egoshooter. Berlin: Ch. Links Verlag, S. 7-25.

Blättel-Mink, Birgit/Hellmann, Kai-Uwe (Hgg.) (2010): Prosumer Revisited. Zur Aktualität einer Debatte. Wiesbaden: VS Verlag für Sozialwissenschaften. DOI: 10.1007/978-3-531-91998-0.

Bredekamp, Horst (2016): Das Beispiel Palmyra. Köln: Walther König.

Brühl, Jannis/Ernst, Anna (2019): „Facebook: Mehr als 1,5 Millionen TerrorVideos gelöscht.«In: Süddeutsche Zeitung. https://www.sueddeutsche.de/poli tik/facebook-video-christchurch-kopien-1.4374124 (19.03.2019) [letzter Zugriff 14.7.2020].

Butler, Judith (1993): »Endangered/Endangering: Schematic Racism and White Paranoia«. In: Gooding-Williams, Robert (Hg.): Reading Rodney King/Reading Urban Uprising. New York: Routledge, S. 15-22.

»Christchurch Call« (2019): https://www.christchurchcall.com/call.html (15.5.2019) [letzter Zugriff 14.7.2021].

Curtis, Robin (2008): »Immersion und Einfühlung: Zwischen Repräsentationalität und Materialität bewegter Bilder«. In: montage AV. Zeitschrift für Theorie und Geschichte audiovisueller Kommunikation, Jg. 17, Nr. 2, S. 89-107. DOI: 10.25969/mediarep/465.

Dewey, Caitlin (2016): »The (Very) Dark Side of Live-Streaming That No One Seems Able to Stop«. In: Washington Post. https:/www.washingtonpost.com/news/th e-intersect/wp/2016/05/26/the-very-dark-side-of-live-streaming-that-no-oneseems-able-to-stop/ (26.05.2016) [letzter Zugriff 14.7.2021].

Ehrenberg, Markus/Sagatz, Kurt (2016): „Die Inszenierungsmaschine«. In: Der Tagesspiegel. https://www.tagesspiegel.de/gesellschaft/medien/terror-im-livest ream-die-inszenierungsmaschine/13760780.html (20.06.2016) [letzter Zugriff 14.7.2021]. 
Fain, Kimberly (2016): »Viral Black Death: Why We Must Watch Citizen Videos of Police Violence«. In: JSTOR Daily. https://daily.jstor.org/why-we-must-watchcitizen-videos-of-police-violence/. (01.09.2016) [letzter Zugriff 14.7.2021].

Gunthert, André (2019): Das geteilte Bild. Essays zur digitalen Fotografie. Göttingen: Konstanz University Press.

Grau, Oliver (2003): Virtual Art. From Illusion to Immersion. Cambridge: MIT Press. DOI: $10.7551 / \mathrm{mitpress} / 7104.001 .0001$.

Haas, Michaela (2017): »Der Tod von Philando Castile«. In.: SZ Magazin: https://szmagazin.sueddeutsche.de/wild-wild-west-amerikakolumne/warum-philando -castile-sterben-musste-83743 (06.04.2017) [letzter Zugriff 14.7.2021].

Honan, Mat (2016): »Why Facebook and Mark Zuckerberg Went All In On Live Video«. In: BuzzFeed News. https:/www.buzzfeednews.com/article/mathona $\mathrm{n}$ /why-facebook-and-mark-zuckerberg-went-all-in-on-live-video (06.04.2016) [letzter Zugriff 14.7.2021].

Joselit, David (2015): »Material Witness: Visual Evidence and the Case of Eric Garner«. In: Artforum. https://www.artforum.com/print/201502/material-witnessvisual-evidence-and-the-case-of-eric-garner-49798 [letzter Zugriff 14.7.2021]. Juhasz, Alexandra (2016): »How Do I (Not) Look? Live Feed Video and Viral Black Death«. In: JSTOR Daily. https://daily.jstor.org/how-do-i-not-look/ (20.7.2016) [letzter Zugriff 14.7.2021].

Klonk, Charlotte (2017): Terror. Wenn Bilder zu Waffen werden. Berlin: S. Fischer.

Kohout, Annekathrin (2017): »Livestreaming is Life«. In: POP. Kultur und Kritik, Jg.10, Nr. 1, S. 74-77. DOI: 10.14361/pop-2017-0112.

Konzeptgruppe »Invektivität« (Dagmar Ellerbrock, Lars Koch, Sabine Müller-Mall, Marina Münkler, Joachim Scharloth, Dominik Schrage und Gerd Schwerhoff) (2017): »Invektivität - Perspektiven eines neuen Forschungsprogramms in den Kultur- und Sozialwissenschaften«. In: Kulturwissenschaftliche Zeitschrift, Jg. 2, Nr. 1, S. 2-24. DOI: 10.2478/kwg-2017-0001.

Mott, Nathaniel (2016): »Why Lavis Reynolds Posted the Philando Castile Shooting Video to Facebook«. In: Inverse. https://www.inverse.com/article/17978-falconheights-police-shooting-details-facebook [letzter Zugriff 14.7.2021].

Nagle, Angela (2017): Kill All Normies. Online Culture Wars from 4chan and Tumblr to Trump and the Alt-Right. Alresford: Zero Books.

Papailias, Penelope (2019): »Witnessing to Survive. Selfie Videos, Live Mobile Witnessing and Black Necropolitics«. In: Schankweiler, Kerstin/Straub, Verena/Wendl, Tobias (Hgg.): Image Testimonies. Witnessing in Times of Social Media. London: Routledge, S. 104-120. DOI: 10.4324/9780429434853-8.

Pörksen, Bernhard (2019): »Wider die Attentatspornografie $\lll$. In: Deutschlandfunk Kultur. https:/www.deutschlandfunkkultur.de/anschlaege-in-christch urch-wider-die-attentatspornografie.2156.de.html?dram:article_id=443775 (15.03.2019) [letzter Zugriff 14.7.2021]. 
Richardson, Michael/Schankweiler, Kerstin (2018): »Affective Witnessing«. In: Slaby, Jan/Scheve, Christian von (Hgg.): Affective Societies. Key Concepts. London: Routledge, S. 166-177. DOI: 10.4324/9781351039260-14.

Rubin, Alissa J./Blaise, Lilia (2016): »Killing Twice for ISIS and Saying So Live on Facebook«. In: The New York Times. https:/www.nytimes.com/2016/06/15/world /europe/france-stabbing-police-magnanville-isis.html (14.06.2016) [letzter $\mathrm{Zu}$ griff 14.7.2021].

Schankweiler, Kerstin (2019): Bildproteste. Widerstand im Netz. Berlin: Wagenbach.

Schankweiler, Kerstin/Straub, Verena/Wendl, Tobias (Hgg.) (2019): Image Testimonies. Witnessing in Times of Social Media. London: Routledge. DOI: $10.4324 / 9780429434853$.

Sieber, Roland (2020): »Terror als Spiel. Virtuell vernetzter Rechtsterrorismus rund um den Globus«. In: Baeck, Jean-Philipp/Speit, Andreas (Hgg.): Rechte Egoshooter. Von der virtuellen Hetze zum Livestream-Attentat. Berlin: Ch. Links Verlag, S. 4666.

Smith, Shawn Michelle (2000): ")Looking at One's Self through the Eyes of Others«: W.E.B. Du Bois's Photographs for the 1900 Paris Exposition«. In: African American Review, Jg. 34, Nr. 4, S. 581-599. DOI: 10.2307/2901420.

Solanas, Fernando/Getino, Octavio (1970): »Toward a Third Cinema«. In: Cinéaste, Jg. 4, Nr.3, S. 1-10.

Sontag, Susan (2001): On Photography [1977]. New York: Picador.

Sontag, Susan (2003): Regarding the Pain of Others. New York: Picador.

Stelter, Brian (2016): "Philando Castile and the Power of Facebook Live«. In: CNN. https://money.cnn.com/2016/07/07/media/facebook-live-streaming-poli ce-shooting/index.html (07.07.2016) [letzter Zugriff 14.7.2021].

Stern Online (2016): »Islamist zeigte Video der Tat auf Facebook«. In: Stern Online: https:/www.stern.de/politik/ausland/attentat-von-magnanville--islamist -stellte-video-der-tat-auf-facebook-6900008.html. (14.06.2016) [letzter Zugriff 14.7.2021].

Straub, Verena (2021): Das Selbstmordattentat im Bild. Aktualität und Geschichte von Märtyrerzeugnissen. Bielefeld: transcript.

Straub, Verena (2019): »Living Martyrs: Testifying What is to Come«. In: Schankweiler, Kerstin/Straub, Verena/Wendl, Tobias (Hgg.): Image Testimonies. Witnessing in Times of Social Media. London: Routledge, S. 139-153. DOI: 10.4324/9780429434853-10.

Tarabay, Jamie (2019): »As New Zealand Fights Online Hate, the Internet's Darkest Corners Resist«. In: The New York Times. https://www.nytimes.com/2019/07/05/ world/asia/new-zealand-internet.html (05.07.2019) [letzter Zugriff 14.7.2021].

Thompson, Iain (2016): »Facebook >Glitch< That Deleted the Philando Castile Shooting Vid: It Was the Police-Sources«. In: The Register. https://www.theregister. 
com/2016/07/08/castile_shooting_police_deletion/ (08.07.2016) [letzter Zugriff 14.7.2021].

Ullrich, Wolfgang (2019): Selfies. Berlin: Wagenbach.

Venus, Jochen (2018): »Gewalt«. In: Beil, Benjamin/Hensel, Thomas/Rauscher, Andreas (Hgg.): Game Studies. Wiesbaden: Springer, S. 331-342. DOI: 10.1007/9783-658-13498-3_18.

Weissman, Terri (2019): „Whose Streets? Police Violence and the Recorded Image«. In: Arts, Jg. 8, Nr. 4, S. 155. DOI: 10.3390/arts8040155.

Wiegel, Michaela (2016): »Polizistenmord nahe Paris: Einer von 8250 Islamisten«. In: Frankfurter Allgemeine Zeitung. www.faz.net/1.4287226. (14.06.2016) [letzter Zugriff 14.7.2021].

\section{Filmverzeichnis}

RAW FOOTAGE: Philando Castile SHOT **FULL VIDEO** (2016) (Right Now News). https://www.youtube.com/watch?v=K_J3sYIgvUE. (7.7.2016) [letzter Zugriff 14.7.2021].

\section{Abbildungsverzeichnis}

Abb. 1: Filmstill aus dem Videotestament von Larossi Abballa, am 13.6.2016 per Facebook Live verbreitet, $13 \mathrm{~min}$, gekürzte Version abrufbar unter: Allen/Summers/Robinson 2016.

Abb. 2-4: Filmstills aus dem Livestream von Diamond Reynolds, am 6.7.2016 per Facebook Live verbreitet, 9:46 min, abrufbar unter: Right Now News 2016. 